\title{
Sektion Gynäkologie und Geburtshilfe: Neustrukturierung des Mehrstufenkonzepts
}

Die Sektion Gynäkologie und Geburtshilfe hat ihr Mehrstufenkonzept inhaltlich neu strukturiert. Ab 1. Januar 2022 besteht die Möglichkeit, eine DEGUMStufe II für die gynäkologische Sonografie zu beantragen.

Bis dato war die gynäkologische Sonografie in der Stufe II der Sektion Gynäkologie und Geburtshilfe implementiert. Dies entsprach jedoch nicht ihrem gelebten Inhalt und spiegelte sich nicht ausreichend im Zertifizierungsprozess wider. Die bisherige Stufe II legt ihren Schwerpunkt maßgeblich auf die Pränataldiagnostik und Geburtshilfe.

Eine unzweifelhafte Evidenz zur zentralen Bedeutung des transvaginalen Ultraschalls in der präoperativen Dignitätseinschätzung der Adnexe, leitlinienbasierter Einsatz der 3D-Sonografie in der Diagnostik von Müllergang-Anomalien, zunehmender Bedarf an Infertilitätsdiagnostik oder die steigende Inzidenz von Sectio-Narbenimplantationen sind nur wenige exemplarisch genannte Beispiele für die wachsenden Anforderungen an die gynäkologische Sonografie. Zum einen bedarf es gezielter Ausbildungswie auch Qualitätssicherungskonzepte, zum anderen muss es für vorhandene Expert*innen eine Möglichkeit geben, sich als solche auszuzeichnen. Folglich bestand dringender Bedarf einer eigenständigen Stufe II für die gynäkologische Sonografie.
Der Ausarbeitung entsprechender Konzepte hat sich eine Arbeitsgemeinschaft „Gynäkologische Sonografie“ gewidmet. Ein erster Schritt war die Implementierung von gynäkologischen Sonografien bei der Beantragung der Stufe I. Auf dem Niveau der Stufe I bleibt es folglich bei dem kombinierten Erwerb für Gynäkologie und Geburtshilfe, aber die gynäkologischen Inhalte sind im Zertifizierungsprozess seit 2020 neu hinzugekommen.

Für die Qualifikation und Zertifizierung über die Stufe I hinaus ergeben sich zwei Wege: die Stufe II für „Pränatale Sonografie“ und die Stufe II für „Gynäkologische Sonografie“. Der Zertifizierungs- und Rezertifizierungsablauf für die Stufe II „Pränatale Sonografie“ entspricht in seinen Vorgaben (Befunde, Nachweis DEGUM-zertifizierter Fortbildungen usw.) den bisher bekannten Regelungen. Für die Stufe II „Gynäkologische Sonografie“ sind analoge Anforderungen formuliert worden, welche sich maßgeblich auf die Vorlage von operativ/ histologisch bestätigten, sonografischen Befunden und den Nachweis einer Mindestanzahl an Fortbildungen stützen.

Eine simultane Beantragung beider Stufen ist möglich.

Für die bisherigen Inhaber*innen der Stufe II Gynäkologie und Geburtshilfe gilt eine
Übergangsregelung, welche den Bestandsschutz in den Vordergrund stellt. Sie behalten diese Stufenbezeichnung bis zur routinemäßig anstehenden Rezertifizierung. Zu diesem Zeitpunkt müssen sie dann entscheiden, ob sie sich ausschließlich für die Stufe II „Pränatale Sonografie“ oder zusätzlich für die Stufe II „Gynäkologische Sonografie“ rezertifizieren.

Die detaillierten Vorgaben sind auf der DEGUM-Homepage (www.degum.de) hinterlegt. Die Qualitätsanforderungen für die gynäkologische Sonografie wurden von der Arbeitsgruppe in gemeinschaftlicher Kooperation der DEGUM, ÖGUM und SGUM sowohl für die Stufe I als auch für die Stufe II in zwei Publikationen zusammengefasst, welche absehbar für den Ultraschall in der Medizin verfügbar sein werden.

Anstehende Aufgaben der Arbeitsgemeinschaft „Gynäkologische Sonografie“ werden die Ausweitung des bisherigen Ausund Fortbildungsangebotes wie auch die Ausarbeitung einer Kursleiter-Struktur sein.

Wir sehen dem Wachstum dieses neuen Arms der Sektion mit freudiger Erwartung entgegen und sind sicher, dass sich die Familie der DEGUM nun auch um gynäkologisch tätige Kolleginnen und Kollegen erweitern wird, deren Schwerpunkt eher in der (prä-)operativen Gynäkologie liegt. 